\title{
Correction to: Hypofractionated sequential radiotherapy boost: a promising strategy in inoperable locally advanced pancreatic cancer patients
}

\author{
Gian Carlo Mattiucci ${ }^{1,2}$. Luca Boldrini ${ }^{1,2}$ - Alessia Nardangeli ${ }^{2}$. Andrea D'Aviero' ${ }^{1}$ - Milly Buwenge ${ }^{3}$. \\ Francesco Cellini ${ }^{2} \cdot$ Francesco Deodato $^{4} \cdot$ Nicola Dinapoli $^{2}$ - Vincenzo Frascino ${ }^{2} \cdot$ Gabriella Macchia ${ }^{4}$. \\ Alessio Giuseppe Morganti ${ }^{3}$. Vincenzo Valentini ${ }^{1,2}$
}

Published online: 9 November 2020

(c) Springer-Verlag GmbH Germany, part of Springer Nature 2020

\section{Correction to: Journal of Cancer Research and Clinical Oncology https://doi.org/10.1007/s00432-020-03411-7}

In the original article published, the last sentence in the sixth paragraph of the introduction section is incorrect. The correct sentence is "This data, were confirmed also in an Italian phase II study, in which it has been demonstrated that gemcitabine-based chemoradiotherapy was correlated with improved overall survival, especially in pts who are clinically more fit to complete the foreseen treatment schedule (CRT) (Mattiucci et al. 2010)".
The original article has been corrected.

Publisher's Note Springer Nature remains neutral with regard to jurisdictional claims in published maps and institutional affiliations.
The original article can be found online at https://doi.org/10.1007/ s00432-020-03411-7.

Andrea D'Aviero

andreadav89@gmail.com

1 Università Cattolica del Sacro Cuore, Roma, Italy

2 UOC Radioterapia Oncologica, Dipartimento Di Diagnostica Per Immagini, Radioterapia Oncologica ed Ematologia, Fondazione Policlinico Universitario “A. Gemelli” IRCCS, Roma, Italy

3 Radiation Oncology Center, Department of Experimental, Diagnostic and Specialty Medicine (DIMES), University of Bologna, S. Orsola-Malpighi Hospital, Bologna, Italy

4 Unità Operativa Di Radioterapia, Gemelli Molise Hospital, Campobasso, Italy 